\title{
Psychiatric Complications of Celiac Disease
}

\author{
Malgorzata Urban - Kowalczyk ${ }^{*}$ \\ Department of Affective and Psychotic Disorder, Medical University of Lodz, Poland \\ *Corresponding author: malgorzata.urban1@wp.pl
}

Received December 04, 2014; Revised December 14, 2014; Accepted December 17, 2014

\begin{abstract}
Celiac disease can manifest as psychiatric disorders like depression, anxiety or psychosis. Coincidence with eating disorders was also described. Usually proper diagnosis is performed secondary to psychiatric symptoms. In some cases gluten -free diet can reduce or even resolve both gastrointestinal and psychiatric symptoms.
\end{abstract}

Keywords: celiac disease, depression, anxiety, schizophrenia, anorexia nervosa, gluten

Cite This Article: Małgorzata Urban - Kowalczyk, "Psychiatric Complications of Celiac Disease." International Journal of Celiac Disease, vol. 3, no. 1 (2015): 25-27. doi: 10.12691/ijcd-3-1-1.

\section{Introduction}

Celiac disease (CD) is an immune-mediated systemic condition elicited by gluten and related prolamines in genetically predisposed individuals. It is associated to structural and functional alterations in gastrointestinal system. CD may be diagnosed at any age and it can affect many organ systems. Moreover, CD can cause neurological and psychiatric complications in adults and children. The article presents main psychiatric disorders related with $\mathrm{CD}$.

\section{Comorbidity of Mental Disorders}

\subsection{Depression}

Depression can be a manifestation of undiagnosed CD [1]. Some authors found it as more common in CD patients than controls [2,3]. Often described symptoms include apathy, severe anxiety and irritability [4]. The prevalence of depressive symptoms in CD population is estimated at 30-69\% [5,6,7] and depressive disorders at $42 \%$ [2]. Pynnönen et al. [8] found the lifetime prevalence of major depressive disorders in adolescents with $\mathrm{CD}$ at $31 \%$ vs $7 \%$ in controls. The prevalence of double depression was also higher in this population than in controls (21\% vs 0\%). In most cases psychiatric symptoms occurred before intestinal biopsy. Female with CD probably reported more depressive symptoms than male patients [9].

A few risk factors for depression in CD were suggested like low quality of life after diagnosis, difficulty in adapting to the chronic disease and malnutrition [3]. Exact cause of depressive symptoms in celiac disease is unknown. Association with autoimmune thyroid disease, nutritional deficiencies, regional blood flow abnormalities with cerebral hypoperfusion were proposed [10]. Impaired availability of monoamine precursor - tryptophan and disturbance in serotoninergic activity were also hypothesized.
Hernanz and Polanco [11] examined untreated and treated children with CD. They found significantly higher tryptophan concentration in healthy control comparison to CD samples ones. Moreover, tryptophan level in treated patients sample was higher than in untreated. In adults only 4 of the 11 celiac patients showed impaired brain availability with respect to tryptophan [12].

\subsection{Anxiety}

Häuser et al [13] reported that anxiety symptoms are more severe in women with $\mathrm{CD}$ on gluten - free diet in comparison to controls. On the contrary other authors found that anxiety is neither more common nor more severe in patients with CD than in healthy controls [14]. Addolorato et al. [15] evaluate the presence of social phobia in 40 adult CD patients. Symptoms of social phobia were significantly more frequent in CD patients than in controls (70\% vs 16\%) irrespective of gluten-free diet. The presence of gastrointestinal symptoms, fear of being judged as a sick person by others and worry of the urgency to find sanitary facilities that possibly might not be available can induce avoidant behaviors in CD patients. In most studies anxiety is consider as a symptom associated to depressive disorder. Further research investigated various type of anxiety disorder in CD population are required.

\subsection{Psychosis}

Compared with the general population, individuals with schizophrenia have an increased prevalence of some autoimmune diseases and reveal immunological abnormalities which suggest that aberrant autoimmune responses may play a role in the aetiology of schizophrenia [16]. Increased immune sensitivity to gluten has been reported in schizophrenia. In the past it was even suggested that gluten may serve as an environmental trigger for schizophrenia in genetically susceptible individuals. Some authors reported increased coexistence of CD and schizophrenia [17] but other studies have not found an association $[18,19]$. Dickerson 
et al.[20] examined large samples of patient with recentonset psychosis and with multi-episode schizophrenia and reported increased levels of IgG and IgA antibodies to gliadin compared to control subjects. Schizophrenic patients in both samples did not have increased levels of IgG antibodies to deamidated gliadin or IgA antibodies to tissue transglutaminase, which are found in most individuals with CD. Authors concluded that patients with schizophrenia recognize epitopes on gliadin molecules but that the antigenic epitopes recognized differ from those recognized by individuals with CD [21]. Moreover they usually do not present clinical gastrointestinal symptoms characteristic for CD. Schizophrenia and CD share some immune abnormalities like increase levels of interleukin (IL-6, IL-8), interferon- $\gamma$ or monocytes ratio [22]. It can suggest that these both disorders may be heterogeneous presentation of a similar cause.

There is a very small piece of information about CD and bipolar disorder. Dickerson et al. [23] investigated patient with this diagnosis with mild psychiatric symptoms severity. They found increased levels of IgG antibodies to gliadin and also increased levels of antibodies to deamidated gliadin compared with controls. The levels of IgA antigliadin antibodies and antibodies to tissue transglutaminase did not differ significantly between groups. In the next study the authors have assessed levels of antibodies in individuals with severe manic episode and have compared them to antibody levels at a 6-month follow up [24]. The results have indicated that patients with mania have increased levels of IgG antibodies to gliadin as compared to healthy controls. In 6month follow-up, the levels of IgG antibodies in the manic patients were not statistically different from those in the control group. It can be concluded that levels of immune activation as measured by IgG antibodies to gliadin are lower after acute manic episode than in individuals with bipolar disorder in stable mental state.

\subsection{Anorexia Nervosa}

There is barely any information referring to role of the gastrointestinal system in mimicking or induced eating disorders. Coincidences of CD and eating disorders were described mainly in few case reports [25,26,27]. In majority of them the diagnosis of CD was performed after psychiatric diagnosis. Described cases showed that symptoms of these two disorders can overlap. Moreover their coincidence may results in different outcome [27]. Yucel et al. [26] described patient with atypical eating disorder and next CD diagnosis who achieved improvement in both conditions after implementation of proper treatment. In one of presented cases anorexia symptoms were interpreted as misdiagnosis because after confirmation of CD and introduction of dietary treatment all symptoms resolved [27].

The results of one study [28] indicate that adult patients with untreated CD have increased frequency of altered eating behavior in comparison to healthy controls. Nevertheless, CD do not appear to be overrepresented in patients with anorexia nervosa - Basso et al. [29] assessed it prevalence at $0.6 \%$. Therefore the screening for CD is recommended in AN patients in which the symptoms, such as digestive complaints, are only partially responding to psychiatric interventions.
The relationship between $\mathrm{CD}$ and eating disorders is unclear but the presence of CD symptoms may confuse the clinical manifestation of both disorders and hamper the diagnosis and treatment.

\subsection{Gluten - Free Diet in Treatment of Psychiatric Symptoms in CD}

Gluten withdrawal is a basic condition for effective treatment of CD but it is controversial if gluten-free diet in fact improves psychiatric symptoms in CD patients. Pynnönen et al [30] reported improvement in psychiatric symptoms after starting a gluten-free diet. It was associated with decrease in CD activity. In other study improvement in anxiety only but not in depressive symptoms during gluten-free diet was observed [7]. Moreover, no association between depressive symptoms and duration of gluten-free diet was found. Ciacci et al. [6] also reported no correlation between depression and CD duration or dietary compliance. The results of metaanalysis of Smith and Gerdes [14] indicated that glutenfree diet was often unable to ameliorate depressive symptoms in adults with CD.

The efficacy of gluten-free diet in schizophrenia was also analyzed in some reports but the majority of them was performed before 1990 and referred to small samples of patients. The results were contradictory - some authors described significant improvement in mental state and some reported no improvement in symptoms or functioning [31,32,33,34]. DeSantis et al. [35] described a schizophrenic patient with severe diarrhea and weight loss. Introduction of gluten-free diet results rapid improvement in somatic and psychiatric symptoms. SPECT scans taken before and after treatment showed a remarkable resolution of decreased frontal cortical blood flow, which is associated with schizophrenia, after initiation of the diet. In several years follow-up she is in remission of psychotic symptoms. This case indicated that schizophrenia-like symptoms can be psychiatric manifestation of CD.

Similar to other mental disorders there is no univocal opinion of gluten-free diet benefits in patients with eating disorders. Gluten withdrawal improves the intestinal absorption and may induced weight gain. Contradictory it was observed that weight gain during gluten-free diet was associated to increase fear of weight gain and exacerbation of eating disorders [27].

\section{Conclusions}

In some cases of CD no evident gastrointestinal symptoms are observed. Even in symptomatic course of the disease usually there is a long time interval between first clinical signs and clarifying the diagnosis. That is why many patients with prior psychiatric diagnosis have undetected chronic bowel inflammation. Since undiagnosed CD predisposes to mental disorders, great attention should be maintained to exclude this condition in psychiatric patients.

\section{Statement of Competing Interests}

None to declare. 


\section{References}

[1] Cannings-John, R., Butler, C.C., Prout, H. et al. A case control study of presentations in general practice before diagnosis of coeliac disease. Br J Gen Pract. 2007; 57: 636-642.

[2] Carta MG, Hardoy MC, Boi MF, Mariotti S, Carpiniello B, Usai P.Association between panic disorder, major depressive disorder and celiac disease: a possible role of thyroid autoimmunity. J Psychosom Res 2002; 53: 789-793.

[3] Ludvigsson JF, Reutfors J, Osby U, Ekbom A, Montgomery SM. Celiac disease and risk of mood disorders - a general population based cohort study. J Affect Disord 2007; 99: 117-126.

[4] Bushara KO. Neurologic presentation of celiac se. Gastroenterol.2005; 128: 92-97.

[5] Goldberg D. A psychiatric study of patients with diseases of the small intestine. Gut 1970; 11: 459-465.

[6] Ciacci C, Iavarone A, Mazzacca G, DeRosa A. Depressive symptoms in adult coeliac disease. Scand J Gastroenterol. 1998; 33: 247-250.

[7] Addolorato G, Capristo E, Ghittoni G. et al. Anxiety but not depression decreases in coeliac patients after one-year gluten-free diet: a longitudinal study. Scand J Gastroenterol. 2001; 36: 502506.

[8] Pynnönen PA, IsometsäET, Aronen E. et al. Mental disorders in adolescents with celiac disease. Psychosomatics 2004; 45: 325335.

[9] Roos S, Karner A, Hallert C. Psychological well-being of adult coeliac patients treated for 10 years. Dig Liver Dis 2006; 38: 177180.

[10] Addolorato G, Di Giuda D, De Rossi G. et al. Regional cerebral hypoperfusion in patients with celiac disease. Am J Med 2004;/116:/312-7.

[11] Hernanz A, Polanco I. plasma precursor amino acids of central nervous system monoamines in children with coeliac disease. Gut. 1991; 32: 1478-1481.

[12] Hallert C, Måtensson J, Allgé LG. Brain availability of monoamine precursors in adult coeliac disease. Scand $\mathrm{J}$ Gastroenterol. 1982;17(1): 87-89.

[13] Häiser W, Janke KH, Klump B, Gregor M, Hinz A. Anxiety and depression in adult patients with celiac disease on a gluten-free diet. W J Gastroenterol.2010;16(22): 2780-2787.

[14] Smith DF, Gerdes LU. Meta-analysis on anxiety and depression in adult celiac disease. Acta Psychiatr Scand 2012; 125: 189-193

[15] Addolorato G, Mirijello A, D'Angelo C et al. Social phobia in coeliac disease. Scand J Gastroenterol. 2008; 43: 410-415.

[16] Müler N, Schwarz MJ. Immune System and Schizophrenia. Curr Immunol Rev. 2010; 6(3): 213-220.

[17] Eaton W, Mortensen PB, Agerbo E, Byrne M, Mors O, Ewald H. Coeliac disease and schizophrenia: Population based case control study with linkage of Danish national registers. BMJ 2004; 328: 438-439.

[18] West J, Logan RF, Hubbard RB, Card T. Risk of renia in people with coeliac disease, ulcerative colitis and Crohn's disease: A general population-based study. Aliment Pharmacol Ther 2006; 23: 71-74.

[19] Ludvigsson JF, Osby U, Ekbom A, Montgomery SM. Coeliac disease and risk of schizophrenia and other psychosis: A general population cohort study. Scand J Gastroenterol. 2007; 42: 179-185.

[20] Dickerson F, Stallings C, Origoni A et al. Markers of gluten sensitivity and celiac disease in recent-onset psychosis and multiepisode schizophrenia. Biol Psychiatry. 2010;68(1): 100-4.

[21] Samaroo D, Dickerson F, Kasarda DD. et al. Novel immune response to gluten in individuals with schizophrenia. Schizophr Res 2010; 118: 248-255.

[22] Kalaydjian AE, Eaton W, Cascella N, Fasano A. The gluten connection: the association between schizophrenia and celiac disease. Acta Psychiatr Scand. 2006;113(2): 82-90.

[23] Dickerson F, Stallings C, Origoni A. et al. Markers of gluten sensitivity and celiac disease in bipolar disorder. Bipolar Disord. 2011; 13(1): 52-8.

[24] Dickerson F, Stallings C, Origoni A, Vaughan C, Khushalani S, Yolken R. Markers of gluten sensitivity in acute mania: a longitudinal study. Psychiatry Res. 2012; 30: 68-71.

[25] Ricca V, Mannucci E, Calabrò A, Bernardo MD, Cabras PL, Rotella CM. Anorexia nervosa and celiac disease: two case reports. Int J Eat Disord. 2000; 27(1): 119-22.

[26] Yucel B, Ozbey N, Demir K, Polat A, Yager J. Eating disorders and celiac disease: a case report. Int J Eat Disord. 2006; 39(6): 530-2.

[27] Leffler DA, Dennis M, Edwards George JB, Kelly CP. The interaction between eating disorders and celiac disease: an exploration of 10 cases. Eur J Gastroenterol Hepatol. 2007; 19(3): 251-5.

[28] Passananti V, Siniscalchi M, Zingone F et al. Prevalence of eating disorders in adults with celiac disease. Gastroenterol Res Pract.2013: 491657.

[29] Basso MS, Zanna V, Panetta F. et al. Is the screening for celiac disease useful in anorexia nervosa? Eur J Pediatr. 2013; 172(2): 261-3.

[30] Pynnönen PA, IsometsäET,Verkasalo MA et al. Gluten-free diet may alleviate depressive and behavioral symptoms in adolescents with coeliac disease: a prospective follow-up case-series study. BMC Psychiatry. 2005; 5: 14

[31] Singh MM, Kay SR. Wheat gluten as a pathogenic factor in schizophrenia. Science 1976; 191: 401-402.

[32] Rice JR, Ham CH, Gore WE. Another look at gluten in schizophrenia. Am J Psychiatry 1978; 135: 1417-1418.

[33] Potkin SG, Weinberger D, Kleinman $\mathrm{J}$ et al. Wheat gluten challenge in schizophrenic patients. Am J Psychiatry 1981; 138: 1208-1211.

[34] Storms LH, Clopton JM, Wright C. Effects of gluten in schizophrenics. Arch Gen Psychiatry 1982; 39: 323-327.

[35] De Santis A, Addolorato G, Romito A et al. Schizophrenic symptoms and SPECT abnormalities in a coeliac patient: regression after a gluten-free diet. J Intern Med 1997; 242: 421423. 